\title{
The Change of Reticulocyte Count and of Arakawa's Reaction on Administration of Vitamin B* in Lactating Mothers.
}

\author{
94th Report of the Peroxidase Reaction.
}

\author{
By
}

Sh. Shiraishi.

(白石信湢)

(From the Department of Pediatrics, Faculty of Medicine, Töhoku Imperial University, Sendai.

Director: Prof. A. Sato.)

\section{Introduction.}

In my ${ }^{\mathbf{1} 2)}$ preceding paper I reported that mothers secreting milk negative to Arakawa's reaction showed an increase of reticulocyte count and a reticulocyte shift to the left, which was, as I reported, probably an early sign of B-avitaminosis. In the present paper I desire to report the change of the reticulocyte count on an administration of vita$\min \mathrm{B}$.

\section{Method of Experiment.}

1. Counting of reticulocytes (Katsunuma's method ${ }^{33}$ ).

One drop of blood was taken with a sterilized glass rod from the ear-lobe vein of each mother and mingled with $1 \%$ brillant cresil blue (in physiological saline) on an object glass. Then a cover glass was set on it and the reticulocytes were microscopically examined with the oil-immersion objective, and thus the rate of reticulocytes to the total erythrocyte count was determined. Erythrocytes were calculated by use

* Vitamin $B_{1}$ is meant in the present paper.

1) Sh. Shir a is hi, Tohoku J. Exp. Med., 1936, 28, 44.

2) Sh. Shiraishi, Tohoku J. Exp. Med., 1937, 31, 510.

3) S. Katsu numa, Nihon Byori Gakkai Kaishi, 1919, 8, 9. 
of Hayem's solution. The classification of reticulocytes was determined by Heilmeyer's method.")

\section{Testing of Arakawa's reaction. ${ }^{*: * 5)}$}

Milk from each mother was examined with Arakawa's reagent.

\section{Result of Experiment.}

Arakawa's reaction will, as Arakawa's experiment ${ }^{5)}$ shows, change from negative to positive on an administration of vitamin $B$, though the amount of this differs according to cases. This experiment was further confirmed by Taka matsu. ${ }^{6}$ Now, lactating mothers with Arakawa-positive milk seen in daily practice are not acute cases of $\mathrm{B}$-avitaminosis, of course, and a daily administration of vitamin $\mathrm{B}$ in a not very large amount cannot easily make an Arakawa-negative case Arakawa-positive, but when mothers with Arakawa-negative milk are taking vitamin $B$, the milk is losing in its toxicity or its content in a glyoxal-like substance or substances, even if it still remains negative to Araka wa's reaction all the same. This is what was shown by Takamatsu. ${ }^{6}$

In the present work, it was difficult to examine mothers for a long time, because as soon as their infants had recovered from illness they stopped visiting our Dispensary.

Most of the 34 cases given in Table 1 and Table 2 showed an improvement of Arakawa's reaction on a daily administration of vita$\min \mathrm{B}$, and all the casese showed a decrease in the reticulocyte count. In Cases No.4, No. 5, No. 6 and No. 8 in Table 1 and in Cases No. 3, No.4, No. 5, No. 8, No. 11, No. 12, No. 17 and No. 24 in Table 2, Arakawa's reaction did not improve at all, as far as the reaction during the first five minutes was concerned. Yet vitamin B must have decreased ${ }^{6}$ the toxicity of each milk, with the reticulocyte count showing a decreace

4) L. Heilmeyer, Dtsch. Arch. f. klin. Med., 1931, 171, 123.

5) T. Arakawa, Tohoku. J. Exp. Med., 1930, 16, 107.

6) A. Takamats u, Tohoku J. Exp. Med., 1934, 23, 46.

** "Arakawa-positive" may be used in two different senses. One of these is : Arakawa-positive in a biochemical sense. A. sample of human milk is said to have become Arakawa-positive, when it became blue on the addition of Arakawa's reagent. Here it means that the sample was not negative to A ra k a w a's reaction. The other of these tow senses is: Arakawa-positive in a clinical sense. A sample of human milk is clinically Arakawa-positive only when it shows such a reaction as $\#$ or $\#$ in one minute of the addition of the reagent. Another sample of human milk may be Arakawa-positive in the first described sense, but yet clinically negative. 


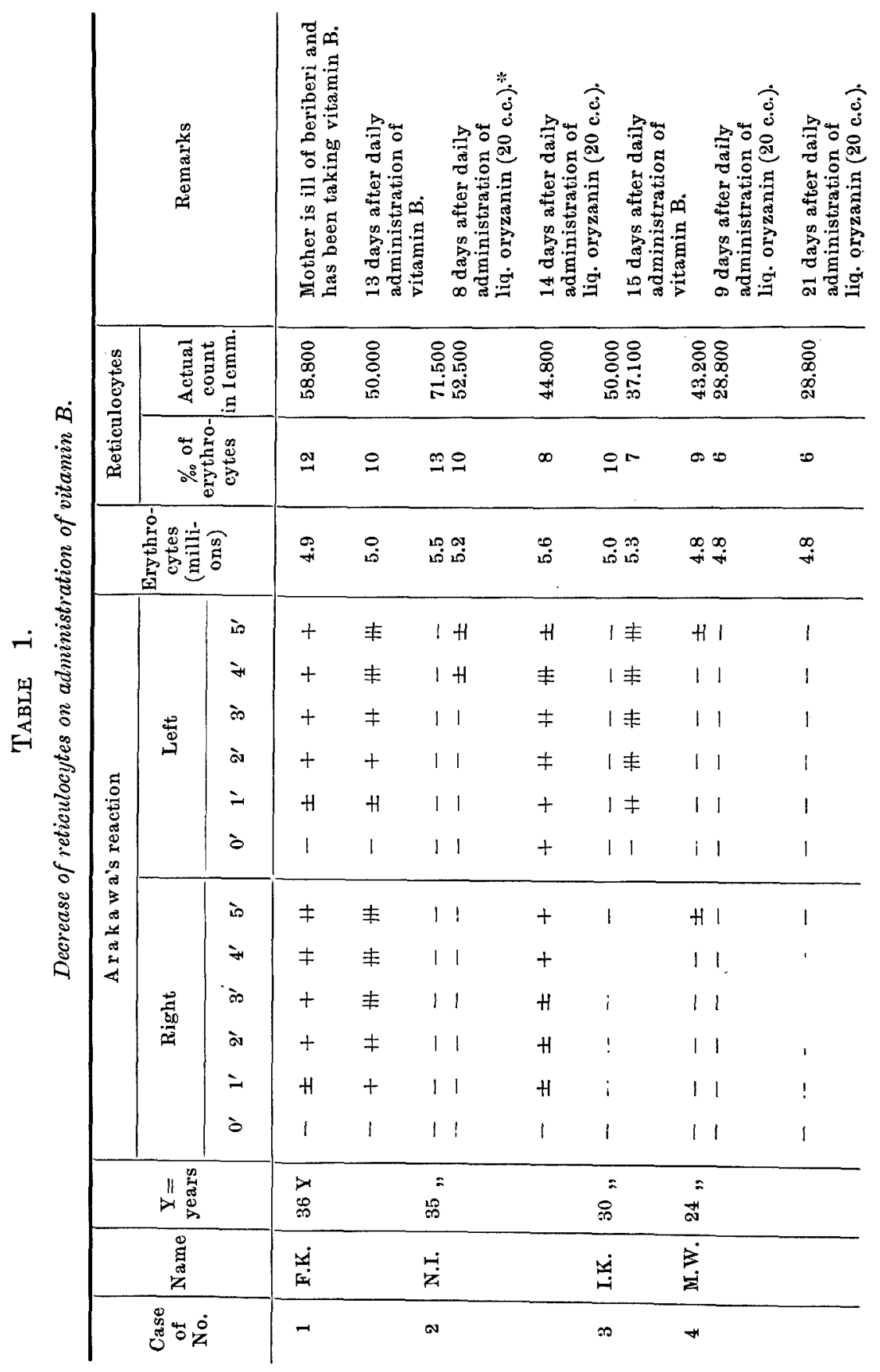




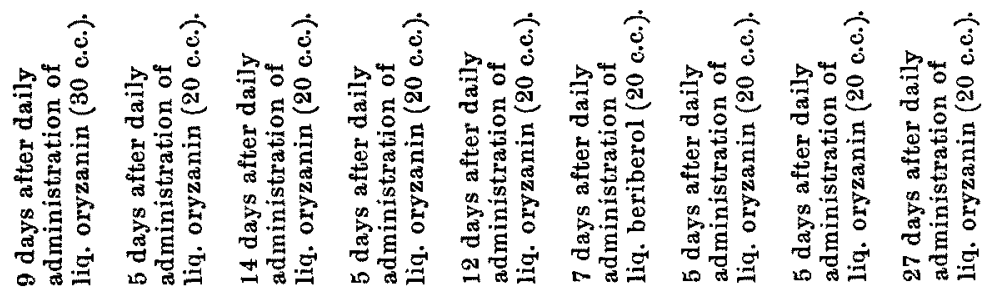

\begin{tabular}{|c|c|c|c|c|c|c|c|c|}
\hline $\begin{array}{l}88 \\
88 \\
80 \\
0 \%\end{array}$ & $\begin{array}{l}8 \% \\
8 \% \\
10 \\
10 \\
0\end{array}$ & $\frac{8}{8}$ & 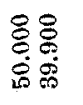 & \begin{tabular}{l}
8 \\
$\stackrel{0}{5}$ \\
\multirow{2}{*}{}
\end{tabular} & $\begin{array}{l}88 \\
88 \\
0 \%\end{array}$ & 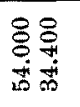 & 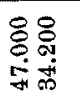 & $\frac{8}{\stackrel{8}{\circ}}$ \\
\hline$\stackrel{\infty}{\sim}$ & $=0$ & $=$ & $\stackrel{n}{\circ}$ & 20 & $m=$ & 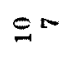 & 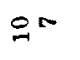 & 10 \\
\hline 0 & 웅 & 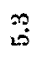 & 05 & 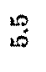 & जि & 牙 & 50 & $\stackrel{\infty}{0}$ \\
\hline$H 1$ & 11 & 1 & $1+$ & $\neq$ & 11 & $+\neq$ & l素 & 羊 \\
\hline 11 & 11 & & $1+$ & $\neq$ & 11 & $+\neq$ & $1 \neq$ & 丰 \\
\hline 11 & 11 & & $1+$ & + & 11 & $H+$ & $1 \neq$ & $\neq$ \\
\hline 11 & 11 & & $1+$ & $\neq$ & 11 & $H+H$ & $1+$ & $\neq$ \\
\hline 11 & 11 & $i$ & 11 & + & $1 i$ & $1+1$ & $1+$ & $\neq$ \\
\hline 11 & 11 & 1 & 11 & 1 & 11 & 11 & 11 & 1 \\
\hline$H 1$ & 11 & 1 & $+\neq$ & $\neq$ & 11 & $1 H$ & 1 丰 & $\neq$ \\
\hline 11 & 11 & & $H \neq$ & $\neq$ & 11 & $1 H$ & | 丰 & 青 \\
\hline 11 & 11 & & $H+$ & $\neq$ & 11 & $1 H$ & | 丰 & 末 \\
\hline 11 & 11 & $i$ & $1+$ & $\mp$ & 11 & 11 & $1 \neq$ & 夆 \\
\hline 11 & 11 & 1 & $1 H$ & + & 11 & 11 & $1+$ & $\neq$ \\
\hline 11 & 11 & 1 & 11 & 1 & 11 & 11 & 11 & 1 \\
\hline$=$ & 5 & & $\stackrel{8}{8}$ & & 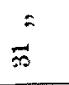 & $\therefore$ & 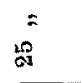 & \\
\hline 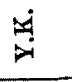 & EH & & Щ्ं & & مُ & $\dot{\infty}$ & ت & \\
\hline
\end{tabular}


Table

Decrease of reticulocyte count and restoration of

\begin{tabular}{|c|c|c|c|c|c|c|c|c|c|c|c|c|c|c|c|}
\hline \multirow{2}{*}{$\begin{array}{c}\text { Case } \\
\text { of } \\
\text { No. }\end{array}$} & \multirow{2}{*}{ Name } & \multirow{2}{*}{$\begin{array}{c}\mathrm{Y}= \\
\text { years }\end{array}$} & \multicolumn{12}{|c|}{ Arakawa's reaction } & \multirow{2}{*}{\begin{tabular}{|c} 
Ery- \\
thro- \\
cytes \\
(milli- \\
ons) \\
\end{tabular}} \\
\hline & & & $0^{\prime}$ & $\mathbf{1}^{\prime}$ & $\begin{array}{l}\mathrm{Ri} \xi \\
2^{\prime}\end{array}$ & & $4^{\prime}$ & $5^{\prime}$ & $0^{\prime}$ & $1^{\prime}$ & $\underset{\mathbf{2}^{\prime}}{\mathrm{Le}}$ & $3^{\prime}$ & $4^{\prime}$ & $5^{\prime}$ & \\
\hline 1 & A.T. & $25 \mathrm{Y}$ & $\bar{z}$ & \pm & $\begin{array}{l}+ \\
\pm\end{array}$ & $\begin{array}{l}+ \\
\pm\end{array}$ & + & + & $=$ & \pm & $\begin{array}{l}+ \\
\pm\end{array}$ & $\begin{array}{l}+ \\
\pm\end{array}$ & + & + & $\begin{array}{l}5.1 \\
5.1\end{array}$ \\
\hline & & & - & \pm & + & H & 世 & 州 & - & \pm & + & $H$ & m & \# & 5.1 \\
\hline 2 & х.т. & $31 "$ & $\begin{array}{l}- \\
-\end{array}$ & $=$ & \pm & + & + & $\begin{array}{l}+ \\
\pm\end{array}$ & - & $\overline{ \pm}$ & $\begin{array}{l} \pm \\
\pm\end{array}$ & $\begin{array}{l}+ \\
\pm\end{array}$ & $\begin{array}{l}+ \\
\pm\end{array}$ & + & $\begin{array}{l}5.2 \\
5.4\end{array}$ \\
\hline 3 & T.S. & $35 "$ & - & - & - & - & \pm & + & - & \pm & + & + & $H$ & $H$ & 4.9 \\
\hline & & & - & - & - & \pm & \pm & \pm & - & \pm & \pm & + & + & + & 5.0 \\
\hline 4 & I.S. & $32 "$ & $\frac{-}{-}$ & $\frac{-}{-}$ & $\frac{-}{-}$ & $\overline{-}$ & $\overline{-}$ & \pm & $=$ & - & $\overline{-}$ & - & $\overline{-}$ & $=$ & $\begin{array}{l}5.3 \\
5.3\end{array}$ \\
\hline 5 & I.0. & $27 "$ & - & - & - & - & - & - & $\overline{-}$ & - & - & 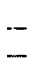 & $=$ & $=$ & $\begin{array}{l}4.2 \\
4.7\end{array}$ \\
\hline 6 & I.K. & 34, & $\begin{array}{l} \pm \\
\pm\end{array}$ & H & $\begin{array}{l}\text { H } \\
H\end{array}$ & $\begin{array}{l}\text { \# } \\
\text { H }\end{array}$ & $\begin{array}{l}\text { \# } \\
\text { H }\end{array}$ & H & $\overline{-}$ & $\overline{ \pm}$ & \pm & $\frac{ \pm}{H}$ & $\frac{ \pm}{H}$ & 声 & $\begin{array}{l}4.5 \\
4.6\end{array}$ \\
\hline 7 & K.I. & 25, & - & - & - & \pm & \pm & \pm & - & - & - & \pm & \pm & \pm & 4.6 \\
\hline & & & - & -- & - & - & - & - & - & \pm & \pm & + & + & + & 4.6 \\
\hline 8 & Y.H. & $29 "$ & $=$ & - & $\underline{-}$ & $\overline{-}$ & $\overline{-}$ & $=$ & $=$ & $=$ & $=$ & $=$ & $=$ & $=$ & $\begin{array}{l}4.9 \\
5.5\end{array}$ \\
\hline 9 & K.K. & $29 "$ & $\overline{-}$ & $\begin{array}{l} \pm \\
\pm\end{array}$ & $\begin{array}{l} \pm \\
\pm\end{array}$ & $\begin{array}{l} \pm \\
+\end{array}$ & $\begin{array}{l} \pm \\
+\end{array}$ & $\frac{ \pm}{H}$ & $\overline{-}$ & $\overline{-}$ & $\begin{array}{l}\overline{ \pm} \\
\pm\end{array}$ & $\begin{array}{l}- \\
\pm\end{array}$ & $\overline{ \pm}$ & $\overline{ \pm}$ & $\begin{array}{l}4.3 \\
4.3\end{array}$ \\
\hline & & & \pm & $H$ & H & H & H & $H$ & - & + & $H$ & 世 & 世 & \# & 4.6 \\
\hline 10 & T.A. & $21 "$ & $\overline{-}$ & - & $=$ & $=$ & - & $\overline{-}$ & $\overline{-}$ & $=$ & - & $\overline{-}$ & $\overline{-}$ & $\overline{ \pm}$ & $\begin{array}{l}4.0 \\
4.5\end{array}$ \\
\hline
\end{tabular}


2.

reticulocyte shift on administration of vitamin $B$.

\begin{tabular}{|c|c|c|c|c|c|c|c|}
\hline \multicolumn{6}{|c|}{ Reticulocytes per mille } & \multirow{2}{*}{$\begin{array}{l}\text { Reticulo- } \\
\text { eytes } \\
\text { (absolute } \\
\text { count) }\end{array}$} & \multirow{2}{*}{ Remarks } \\
\hline 0 & I & II & III & IV & Total & & \\
\hline 0 & 1 & 7 & 6 & 2 & 16 & 81.600 & \\
\hline 0 & 0 & 6 & 10 & 0 & 16 & 81.600 & $\begin{array}{l}4 \text { days after daily administration of liq. } \\
\text { oryzanin* }(20 \text { e.c.) and liq. paranutrin } \\
(20 \text { c.c. }) \text {; and injection of oryzanin for- } \\
\text { tior** (1 c.c.). }\end{array}$ \\
\hline 0 & 0 & 2 & 6 & 0 & 8 & 40.800 & $\begin{array}{l}9 \text { days after daily administration of liq. } \\
\text { oryzanin }(20 \text { c.c.) and liq. paranutrin } \\
(20 \text { c.c.); and injection of oryzanin for- } \\
\text { tior }(1 \text { c.c.) or beriberol }(3 \text { c.c.) and since } \\
5 \text { days injection of yakriton }(0.5 \text { c.c.) } \\
\text { every day. }\end{array}$ \\
\hline 0 & 0 & 6 & 4.5 & 1 & 11.5 & 59.800 & \\
\hline 0 & 0 & 2 & 3 & 1 & 6 & 32.400 & $\begin{array}{l}6 \text { days after daily administration of liq. } \\
\text { oryzanin ( } 30 \text { c.c.) and injection of yak- } \\
\text { riton }(0.5 \text { c.c.). }\end{array}$ \\
\hline 0 & 0 & 3.5 & 2.5 & 1.5 & 7.5 & 36.800 & Has been taking vitamin $B$. \\
\hline 0 & 0 & 0 & 1 & 4 & 5 & 25.000 & $\begin{array}{l}\text { Has been taking vitamin } B \text { for a long } \\
\text { time. }\end{array}$ \\
\hline 0 & 0.5 & 6.5 & 5.5 & 0.5 & 13 & 68.900 & \\
\hline 0 & 0 & 3 & 6 & 0 & 9 & 47.700 & $\begin{array}{l}9 \text { days after daily administration of liq. } \\
\text { oryzanin ( } 20 \text { c.c.). }\end{array}$ \\
\hline 0 & 0 & 9 & 3 & 2 & 14 & 58.800 & \\
\hline 0 & 0 & 2 & 4 & 1 & 7 & 32.900 & $\begin{array}{l}7 \text { days after daily administration of vi- } \\
\text { tamin } B \text {. }\end{array}$ \\
\hline 0 & 0 & 1 & 3 & 1 & 5 & 22.500 & \\
\hline 0 & 0 & 0.5 & 2.5 & 0.5 & 3.5 & 15.800 & $\begin{array}{l}10 \text { days after daily administration of vi- } \\
\text { tamin } B \text {. }\end{array}$ \\
\hline 0 & 0 & 5 & 2.5 & 0.5 & 8 & 26.800 & $\begin{array}{l}\text { Has been taking vitamin } B \text { and injection } \\
\text { of vitamin } B \text { preparation for a long time. }\end{array}$ \\
\hline 0 & 0 & 2 & 2 & 1 & 5 & 23.000 & $\begin{array}{l}22 \text { days after daily administration of vi- } \\
\text { tamin } B \text { and injection of vitamin } B \text { pre- } \\
\text { paration and injection of yakriton ( } 0.5 \\
\text { c.c.). }\end{array}$ \\
\hline 0 & 0 & 7 & 5 & 1 & 13 & 63.700 & \\
\hline 0 & 0 & 2.5 & 3.5 & 1.5 & 7.5 & 41.300 & $\begin{array}{l}7 \text { days after daily administration of liq. } \\
\text { oryzanin }(20 \text { c.c. }) \text {. }\end{array}$ \\
\hline 0 & 0 & 6 & 6 & 0 & 12 & 51.600 & \\
\hline 0 & 0 & 4 & 5 & 1 & 10 & 43.000 & $\begin{array}{l}11 \text { days after daily administration of liq. } \\
\text { oryzanin ( } 20 \text { c.c.). }\end{array}$ \\
\hline 0 & 0 & 3 & 3.5 & 0.5 & 7 & 32.200 & $\begin{array}{l}22 \text { days after daily administration of liq. } \\
\text { oryzanin }(20 \mathrm{c.c} \text {.). }\end{array}$ \\
\hline 0 & 0 & 9 & 9 & 1 & 19 & 76.000 & \\
\hline 0 & 0 & 5.5 & 7.5 & 1 & 14 & 63.000 & $\begin{array}{l}9 \text { days after daily adiministration of vl- } \\
\text { tamin } B \text {. }\end{array}$ \\
\hline
\end{tabular}




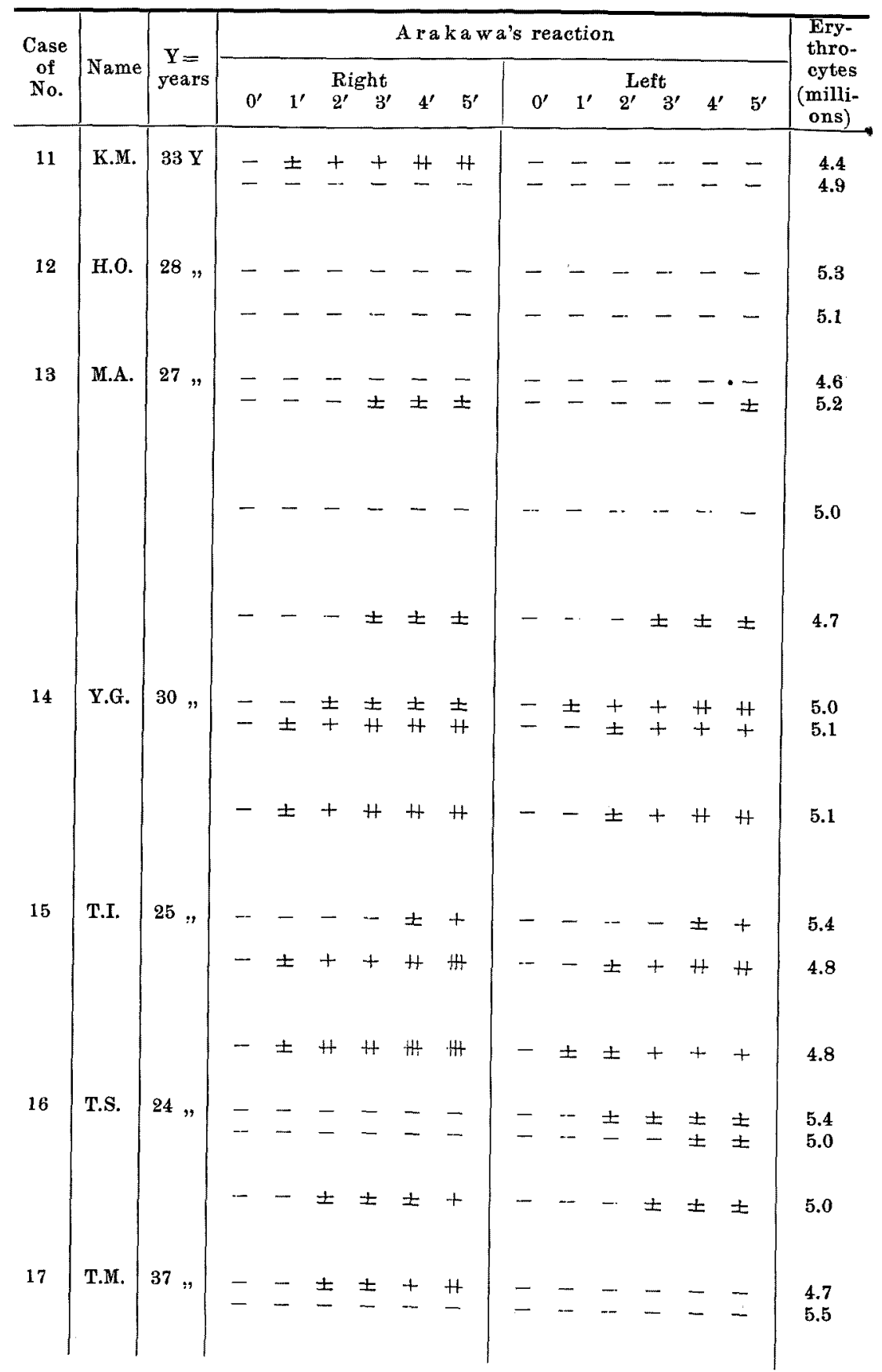




\begin{tabular}{|c|c|c|c|c|c|c|c|}
\hline \multicolumn{6}{|c|}{ Reticulocytes per mille } & \multirow{2}{*}{$\begin{array}{l}\text { Reticulo- } \\
\text { cytes } \\
\text { (absolute } \\
\text { count) }\end{array}$} & \multirow[b]{2}{*}{ Remarks } \\
\hline $\mathbf{0}$ & $\mathbf{I}$ & II & III & IV & Total & & \\
\hline $\mathbf{0}$ & 0 & 6 & 5 & 2 & 13 & 57.200 & \\
\hline 0 & 0 & 5.5 & 5.5 & 1 & 12 & 58.800 & $\begin{array}{l}5 \text { days after daily administration of liq. } \\
\text { oryzanin ( } 20 \text { c.c.) and liq. beriberol }(20 \\
\text { c.c.); and injection of yakriton ( } 0.5 \text { c.c.). }\end{array}$ \\
\hline 0 & 0.5 & 8.5 & 5 & 0.5 & 14.5 & 76.900 & Has been taking vitamin B sometimes. \\
\hline 0 & 0 & 4 & 4.5 & 0.5 & 9 & 45.900 & $\begin{array}{l}\text { Has been taking vitamin } B \text { for a long } \\
\text { time and sometimes injection of oryza- } \\
\text { nin fortior }(1 \text { c.c.). }\end{array}$ \\
\hline 0 & 0 & 4.5 & 3 & 0.5 & 8 & 36.800 & \\
\hline 0 & 0 & 4 & 4 & 0 & 8 & 40.600 & $\begin{array}{l}4 \text { days after daily administration of liq. } \\
\text { oryzanin }(20 \text { c.c.) and injection of ory- } \\
\text { zanin }(1 \text { c.c.). } \text { * } \text { tond injection of yakri- } \\
\text { ton }(0.5 \text { c.c.). }\end{array}$ \\
\hline 0 & 0 & 3.5 & 4.5 & $\mathbf{0}$ & 8 & 40.000 & $\begin{array}{l}11 \text { days after daily administration of liq. } \\
\text { oryzanin ( } 20 \text { c.c.) and injection of ory- } \\
\text { zanin }(1 \text { c.c.) and injection of yakriton } \\
(0.5 \text { c.c.). }\end{array}$ \\
\hline 0 & 0 & 3 & 3.5 & 0.5 & 7 & 32.900 & $\begin{array}{l}20 \text { days after daily administration of liq. } \\
\text { oryzanin }(20 \text { c.c.) and injection of ory- } \\
\text { zanin }(1 \text { c.c.) and injection of yakriton } \\
(0.5 \text { c.c.). }\end{array}$ \\
\hline 0 & 0.5 & 9 & 4.5 & 0 & 14 & 70.000 & \\
\hline 0 & 0 & 5.5 & 4.5 & 0.5 & 10.5 & 53.600 & $\begin{array}{l}5 \text { days after daily administration of liq. } \\
\text { oryzanin }(20 \text { c.c. }) \text { and injection of yak- } \\
\text { riton }(0.5 \text { c.c.). }\end{array}$ \\
\hline 0 & 0 & 2.5 & 2.5 & 1 & 6 & 30.600 & $\begin{array}{l}10 \text { days after daily administration of liq. } \\
\text { oryzanin }(20 \text { c.c.) and injection of yak- } \\
\text { riton }(0.5 \text { c.c.) and since } 5 \text { days injection } \\
\text { of paranutrin ( } 3 \text { c.c.). }\end{array}$ \\
\hline 0 & 0.5 & 5.5 & 4 & 0 & 10 & 54.000 & Has been taking vitamin $B$. \\
\hline 0 & 0 & 4.5 & 5.5 & 1 & 11 & 52.800 & $\begin{array}{l}10 \text { days after daily administration of liq. } \\
\text { oryzanin }(20 \text { c.c.) and injection of yak- } \\
\text { riton }(0.5 \text { c.c. }) \text {. }\end{array}$ \\
\hline 0 & 0 & 3 & 4.5 & 0.5 & 8 & 38.400 & $\begin{array}{l}16 \text { days after daily administration of liq. } \\
\text { oryzanin ( } 20 \text { c.c.) and injection of yak- } \\
\text { riton }(0.5 \text { c.c.). }\end{array}$ \\
\hline 0 & 1 & 9.5 & 4 & 0 & 14.5 & 78.300 & \\
\hline 0 & 0 & 6 & 4 & 0 & 10 & 50.000 & $\begin{array}{l}8 \text { days after daily administration of liq. } \\
\text { oryzanin ( } 20 \text { c.c.). }\end{array}$ \\
\hline 0 & 0 & 3.5 & 4 & 1 & 8.5 & 42.500 & $\begin{array}{l}14 \text { days after daily administration of liq. } \\
\text { oryzanin ( } 20 \text { c.c.) and injection of yak- } \\
\text { riton }(0.5 \text { c.c.) and since } 6 \text { days injection } \\
\text { oryzanin fortior }(1 \text { c.c.). }\end{array}$ \\
\hline 0 & 0 & 7.5 & 5 & 0 & 12.5 & 58.800 & \\
\hline 0 & 0 & 4.5 & 5.5 & 0.5 & 10.5 & 57.800 & $\begin{array}{l}7 \text { days after daily administration of liq. } \\
\text { paranutrin }(10 \text { c.c.) and liq. beriberol } \\
(10 \text { c.c.). }\end{array}$ \\
\hline
\end{tabular}




\begin{tabular}{|c|c|c|c|c|c|c|c|c|c|c|c|c|c|c|c|}
\hline \multirow{2}{*}{$\begin{array}{c}\text { Case } \\
\text { of } \\
\text { No. }\end{array}$} & \multirow[b]{2}{*}{ Name } & \multirow{2}{*}{$\begin{array}{l}\mathrm{Y}= \\
\text { years }\end{array}$} & \multicolumn{12}{|c|}{ Arakawa's reaction } & \multirow{2}{*}{$\begin{array}{c}\text { Ery- } \\
\text { thro- } \\
\text { cytes } \\
\text { (milli- } \\
\text { ons) }\end{array}$} \\
\hline & & & $0^{\prime}$ & $1^{\prime}$ & $\begin{array}{l}\text { Ri } \\
2^{\prime}\end{array}$ & ht & $4^{\prime}$ & $\mathbf{5}^{\prime}$ & $0^{\prime}$ & $1^{\prime}$ & $\underset{2^{\prime}}{\mathrm{L}}$ & $\mathrm{ft}^{\prime}$ & $4^{\prime}$ & $\mathbf{5}^{\prime}$ & \\
\hline 18 & K.Y. & $34 \mathrm{Y}$ & - & - & \pm & \pm & \pm & \pm & $\overline{-}$ & $\begin{array}{l} \pm \\
\pm\end{array}$ & $\begin{array}{l}+ \\
\pm\end{array}$ & + & + & H & $\begin{array}{l}5.1 \\
4.9\end{array}$ \\
\hline & & & - & - & - & - & \pm & \pm & \pm & + & + & $H$ & $H$ & $H$ & 4.9 \\
\hline 19 & T.A. & 27 & - & - & $\overrightarrow{ \pm}$ & \pm & $\begin{array}{l} \pm \\
+\end{array}$ & $\begin{array}{l} \pm \\
+\end{array}$ & - & - & - & $\overline{ \pm}$ & $\overline{ \pm}$ & $\overline{+}$ & $\begin{array}{l}4.6 \\
4.8\end{array}$ \\
\hline 20 & I.K. & 25, & - & - & \pm & $\overline{ \pm}$ & $\overline{ \pm}$ & $\overline{ \pm}$ & - & $\overline{-}$ & $\overline{ \pm}$ & $\overline{ \pm}$ & $\overline{-}$ & $\overline{ \pm}$ & $\begin{array}{l}4.9 \\
4.9\end{array}$ \\
\hline & & & - & \pm & \pm & + & + & $H$ & - & - & \pm & \pm & \pm & + & 4.9 \\
\hline 21 & T.N. & 30 & $\overline{ \pm}$ & $\overline{+}$ & $\bar{H}$ & $\bar{H}$ & 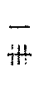 & $\frac{ \pm}{H}$ & - & $\overline{ \pm}$ & $\overline{ \pm}$ & \pm & $\overline{-}$ & \pm & $\begin{array}{l}4.8 \\
5.2\end{array}$ \\
\hline 22 & M.A. & $30 "$ & - & - & \pm & + & + & + & - & - & - & - & - & \pm & 5.8 \\
\hline & & & - & \pm & + & + & + & $H$ & - & \pm & + & + & $H$ & $H$ & 5.6 \\
\hline 23 & T.W. & 34, & - & \pm & + & + & + & + & - & \pm & + & + & + & + & 5.0 \\
\hline & & & - & - & \pm & + & $H$ & $H$ & - & - & \pm & \pm & + & $H$ & 5.0 \\
\hline & & & - & \pm & + & $H$ & $H$ & $H$ & - & \pm & + & $H$ & $H$ & $H$ & 4.6 \\
\hline 24 & T.K. & $25 n$ & - & - & - & - & - & $\overline{-}$ & - & - & \pm & \pm & H & H & $\begin{array}{l}4.3 \\
4.1\end{array}$ \\
\hline & & & - & - & - & - & - & - & - & - & - & - & - & - & 4.2 \\
\hline & * & Liq. or & $\min$ & San & kyc & 10 & $=\mathrm{v}$ & $\operatorname{tam}$ & B, cl & ime & to & onts & in 6 & Inte & national \\
\hline & 并 & Oryzani & fort & or ( & ank & yo) & & (inj & ion) & $=$ vit & $\operatorname{ami}$ & $\mathrm{B}$, & lain & ed $t$ & contain \\
\hline & 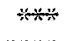 & Oryzani & (Sar & kyo & $1 \mathrm{c}$ & c. $(i$ & iject & (on) & ritan & $\operatorname{in} \mathrm{B}$ & 33 & Inte & rnat & lona & Units in \\
\hline & $\because * * *$ & Oryzani & (Sar & kyo & $1 \mathrm{gl}$ & $\mathrm{m} .=$ & vita & $\min$ & clai & ned & $\mathrm{occ}$ & ntai & n 40 & Inte & enational \\
\hline
\end{tabular}




\begin{tabular}{|c|c|c|c|c|c|c|c|}
\hline \multicolumn{6}{|c|}{ Reticulocytes yer mille } & \multirow{2}{*}{$\begin{array}{l}\text { Reticulo- } \\
\text { cytes } \\
\text { (absolute } \\
\text { count) }\end{array}$} & \multirow{2}{*}{ Remarks } \\
\hline 0 & I & II & III & IV & Total & & \\
\hline 0 & 0 & 5.5 & 2 & 0.5 & 8 & 40.800 & \\
\hline 0 & 0 & 2 & 3.5 & 0.5 & 6 & 29.400 & $\begin{array}{l}7 \text { days after daily administration of liq. } \\
\text { paranutrin }(20 \text { c.c.). }\end{array}$ \\
\hline 0 & 0 & 1 & 2 & 0 & 3 & 14.700 & $\begin{array}{l}14 \text { days after daily administration of liq. } \\
\text { paranutrin ( } 20 \text { c.c.) and since } 7 \text { days in- } \\
\text { jection of yakriton ( } 0.5 \text { c.c.) every other } \\
\text { day. }\end{array}$ \\
\hline 0 & 0 & 6 & 4 & 0 & 10 & 46.000 & \\
\hline 0 & 0 & 1.5 & 3 & 0.5 & 5 & 24.000 & $\begin{array}{l}7 \text { days after daily administration of vi- } \\
\text { tamin } B \text {. }\end{array}$ \\
\hline 0 & 1 & 5 & 4 & 0.5 & 10.5 & 51.500 & \\
\hline 0 & 0 & 2.5 & 3.5 & 0.5 & 6.5 & 31.900 & $\begin{array}{l}5 \text { days after daily administration of liq. } \\
\text { paranutrin }(20 \text { c.c.). }\end{array}$ \\
\hline 0 & 0 & 1 & 3 & 2 & 6 & 29.400 & $\begin{array}{l}14 \text { days after daily administration of liq. } \\
\text { paranutrin }(20 \text { c.c.) and } 8 \text { days after dai- } \\
\text { ly injection of beriberol }(5.0 \text { c.c. }) \text { and } \\
\text { injection of yakriton }(0.5 \text { c.c.). }\end{array}$ \\
\hline 0 & 0 & 4.5 & 4 & 1 & 9.5 & 45.600 & \\
\hline 0 & 0 & 2 & 4 & 1 & 7 & 36.400 & $\begin{array}{l}6 \text { days after daily administration of liq. } \\
\text { oryzanin ( } 20 \text { c.c.) and liq. beriberol ( } 20 \\
\text { c.c.); and injection of yakriton ( } 0.5 \text { c.c.). }\end{array}$ \\
\hline 0 & 0.5 & 6 & 4 & 1 & 11.5 & 66.700 & $\begin{array}{l}\text { Since yesterday administration of liq. } \\
\text { oryzanin }(20 \text { c.c. }) \text { and injection of spel- } \\
\text { zon }(5 \text { c.c. }) \text {. }\end{array}$ \\
\hline 0 & 0 & 2 & 3 & 1.5 & 6.5 & 36.400 & $\begin{array}{l}8 \text { days after daily administration of liq. } \\
\text { oryzanin }(20 \text { c.c.) and injection of spel- } \\
\text { zon }(5.0 \text { c.c.). }\end{array}$ \\
\hline 0 & 1 & 8 & 8 & 1 & 18 & 90.000 & $\begin{array}{l}10 \text { days after daily administration of } \\
\text { oryzanin }(6 \text { grms.). }\end{array}$ \\
\hline 0 & 0.5 & 5 & 5.5 & 1.5 & 12.5 & 62.500 & $\begin{array}{l}16 \text { days after daily administration of } \\
\text { oryzanin ( } 12 \text { grms.). }\end{array}$ \\
\hline 0 & 0 & 5 & 5 & 2 & 12 & 55.200 & $\begin{array}{l}1 \text { month after daily administration of } \\
\text { oryzanin ( } 12 \text { grams.). }\end{array}$ \\
\hline 0 & 1 & 6 & 5 & 2 & 14 & 60.200 & \\
\hline 0 & 0 & 2.5 & 4.5 & 1 & 8 & 32.800 & $\begin{array}{l}1 \text { month after daily administration of } \\
\text { oryzanin ( } 5 \text { grms.) and ebios } \\
\text { grms.) and injection of yakriton ( } 0.5 \text { c.c.) } \\
\text { every other day. }\end{array}$ \\
\hline 0 & 0 & 2 & 3 & 1.5 & 6.5 & 27.300 & $\begin{array}{l}7 \text { days after daily administration of ory- } \\
\text { zanin ( } 5 \text { grms.) and ebios ( } 3.0 \text { grms.) and } \\
\text { injection of yakriton }(0.5 \text { c.c.) and ory- } \\
\text { zanin fortior ( } 1 \text { c.c.) every other day. }\end{array}$ \\
\hline
\end{tabular}

Units in it then.

333 International Units in it then. it then.

Units in it then.

Units in it then. 
too. Table 2 shows that the reticulocyte count decreased on an administration of vitamin $B$ and the reticulocyte shift to the left was made nearer and nearer the normal.

\section{Summary.}

In my preceding paper ${ }^{12)} I$ asserted that mothers with Arakawanegative milk are already in a state of avitaminosis $B$. In the present paper I was able to make the reticulocyte count smaller in mothers of negative or weakly Arakawa's reaction by an administration of vitamin $B$ to them, which fact shows again that Arakawa-negative cases are already in a state of B-avitaminosis.

\section{Conclusion.}

Mothers with weakly or negative Arakawa's reaction show a decrease of reticulocyte court and a restoration of reticulocyte shift to the normal on an administration of vitamin B. The abnormity of the reticulocyte picture in such lactating mothers is due to a state of $\mathrm{B}$-avitaminosis. 